\title{
Herschel observations of NGC 7027
}

\author{
Katrina M. Exter ${ }^{1}$, Peter A.M van Hoof ${ }^{2}$, Griet C. Van de Steene ${ }^{2}$, \\ Roland Ottensamer ${ }^{3}$, Roger Wesson ${ }^{4}$, Mike J. Barlow $^{4}$, Gary J. \\ Ferland $^{5}$, Ryan L. Porter ${ }^{6}$ and the MESS consortium \\ ${ }^{1}$ IvS, Katholieke Universiteit Leuven, Celestijnenlaan 200D, B-3001 Leuven, Beglium \\ ${ }^{2}$ Royal Observatory of Belgium, Ringlaan 3, B-1180 Brussels, Belgium \\ ${ }^{3}$ University Vienna, Dept. of Astronomy, Türkenschanzstrasse 17, A-1180 Wien, Austria \\ ${ }^{4}$ Dept. of Physics and Astronomy, Univ. College London, Gower St, London WC1E 6BT, UK \\ ${ }^{5}$ Dept. of Physics and Astronomy, Univ. of Kentucky, Lexington, KY 40506, USA \\ ${ }^{6}$ Physics and Astronomy and center for Simulational Physics, Univ. of Georgia, Athens, GA \\ 30602-2451, USA
}

\begin{abstract}
We present our analysis of the spectra of NGC 7027 taken with the PACS and SPIRE instruments of the Herschel satellite.
\end{abstract}

Keywords. planetary nebulae: individual (NGC 7027), infrared: ISM

\section{Introduction}

As part of the Herschel Guaranteed Time Key Project MESS (Mass loss of Evolved StarS) (PI Martin Groenewegen) we have gotten (will get) spectra of a sample of planetary nebulae (PNe) with the PACS (Poglitsch et al. 2010) and SPIRE (Griffin et al. 2010) instruments of the Herschel satellite (Pillbratt et al. 2010). A detailed description of the programme can be found in Groenewegen et al. (2011) and an overview of the Herschel observations for PNe in van Hoof et al. (2012).

NGC 7027 is a young bipolar PN with a fairly massive and fast-evolving, hot central star, and a C-rich nebular chemistry. It has an elliptical morphology at most wavelengths. The inner nebula is a prolate spheriodal shell with an equatorial density enhancement, outside of this are various layers of nebula including a larger spherical $\mathrm{CO}$ envelope. In the inner parts, molecular regions interface with the ionised regions with an hourglass morphology, where the wide waist corresponds to the equatorial torus.

\section{The SPIRE data}

The SPIRE spectra of NGC 7027 were presented in Wesson et al. (2010). They detected lines of $\mathrm{CO}, \mathrm{H}_{2} \mathrm{O}$ and $\mathrm{CH}^{+}$, from which they determine a range of rotational temperatures: 50-130 K from CO, 70-260 $\mathrm{K}$ from $\mathrm{CH}^{+}$. This range reflects a complexity in the nebula. Note the presence of water, despite a C-rich chemistry: these result from the PDR being warm and dense, where the harsh radiation field of the central star keep CO partially dissociated.

\section{The PACS data}

The PACS 70- $\mu \mathrm{m}$ image, deconvolved, is shown in Fig. 1. It is a limb-brightened shell, similar (at least qualitatively: an exact comparison still has to be done) to other IR and radio images. 

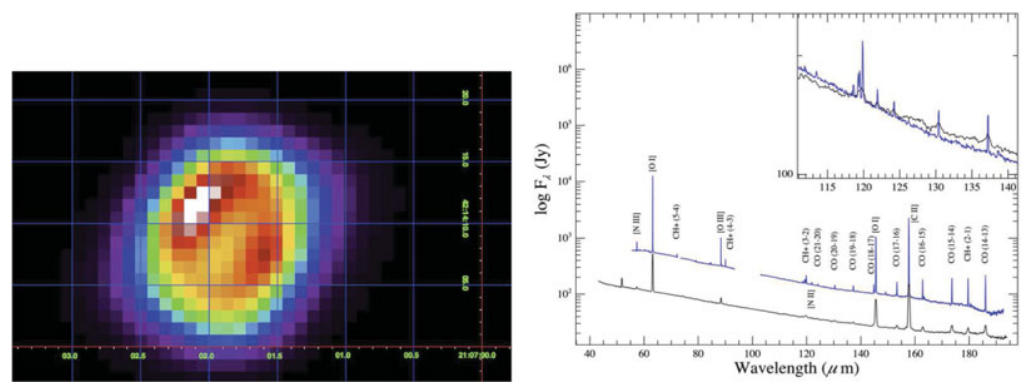

Figure 1. Left: PACS 70- $\mu \mathrm{m}$ image of NGC 7027, deconvolved. Right: PACS spectrum compared to the (lower) offset ISO spectrum. Upper panel compares the PACS and ISO spectra without any offset.

The entire nebula fits inside the centre of the field-of-view of the PACS spectrograph $\left(3 \times 3\right.$ spaxels of $9.4^{\prime \prime}$ each $)$. From these we have extracted a spectrum, which is also presented in Fig. 1. Comparison to the ISO LWS spectrum shows the improvement PACS offers when observing such sources. For a more detailed explanation of the reduction of these data and the production of the spectrum, see a future paper on NGC 7027 (Exter et al., in prep.).

\section{Modelling}

We have a preliminary model of NGC 7027 based on the Herschel data combined with radio, ISO-SWS, near-IR, optical, and UV data, and using the photoionisation/PDR code Cloudy, last described in Ferland et al. (1998). The model was created by varying various input parameters such as elemental abundances, the density of the gas and the dust-togas mass ratio, the inner radius of the circumstellar shell and the effective temperature and luminosity of the central star. The outer radius was determined by reaching the observed CO 12-11 line flux. Since NGC 7027 has a carbon-rich chemistry, we assumed graphite dust and PAHs to be present in the circumstellar shell. We initially tried a constant density model, but found that it could not produce a satisfactory fit to the observations from the ionised region and the PDR. We then tried a constant pressure model, but found that also did not produce a satisfactory fit. We then tried a model where the ionised region and the PDR had a different constant density (except for a narrow region at the ionisation front where there was a continuous change from one density to the other). This produced a good fit. The model indicates a total hydrogen number density of $3.3 \times 10^{4} \mathrm{~cm}^{-3}$ in the ionised region and $7.9 \times 10^{5} \mathrm{~cm}^{-3}$ in the PDR. The most plausible explanation is that when the central star heated up and started to photoionise the circumstellar shell, that part of the shell started to expand. This did not happen in the PDR, or at least the effect was much less there. Also, this process was so fast that to this date the shell has not been able to attain pressure equilibrium.

\section{References}

Ferland, G. J., Korista, K. T., Verner, D. A., et al., 1998, PASP, 110, 761

Griffin, M. J., Abergel, A., Abreu, A., et al., 2010 A\&A, 518, L3

Pilbratt, G. L., Riedinger, J. R., Passvogel, T. et al., 2010, A\& $A$, 518, L1

Poglitsch, A., Waelkens, C., Geis, N. et al., 2010 A $ध A$, 518, L2

van Hoof, P. A. M., Barlow, M. J., Van de Steene, G. C., et al., 2012, these proceedings

Wesson, R., Cernicharo, J., Barlow, M. J., et al., 2010 A\&A, 518, 144 